\section{Information Flow among Developing Cells}

\author{
Cellular Communication during Ocular \\ Development. Cell and Developmental Biology of \\ the Eye (proceedings series). \\ Edited by J. B. Sheffield and S. R. Hilfer.
}

New York: Springer-Verlag. (1982). 196 pp. \$32.50.

Converting a fertilized zygote into a mature adult organism is a complex task requiring exquisite orchestration of events and cooperation among individual cells. Attempts to explain and understand the methods of control and the pathways of communication that exist during differentiation of cells, tissues and organs have become increasingly fruitful as experimental techniques have become more sophisticated. The eye is a uniquely specialized organ with a multitude of physiologically and anatomically distinctive structures all working for a common function: vision. Progress toward understanding how cellular communication contributes to ocular development is the topic of this book.

This volume is the second in a series based on the Proceedings of the Philadelphia Symposia on Ocular and Visual Development. These symposia are traditionally small meetings, not heavily attended by those outside the Philadelphia area, but with a carefully selected roster of invited speakers. The atmosphere is one of informality with an opportunity for the sort of intense and focused exchanges of information and ideas that is often sorely lacking at the large annual gatherings of scientific societies most of us attend.

The 1981 meeting focused on intercellular communication, and this book is a collection of ten papers from that meeting. The contributions are of varying length and scope and, unfortunately, of uneven quality. Some will be most informative to the specialists in a certain field, whereas others will be more generally useful as reviews of the state-of-the-art in their respective subjects. One wishes the editors had exercised more guidance in directing the authors' efforts, to produce a more uniform content. Three main issues are addressed: synaptogenesis and formation of neuronal connections; intercellular communication by coupling of cells during development; and regulation of differentiation by humoral factors. A final paper about the theory of magnifying spectacles and their use by the visually impaired is completely out of place; its inclusion is puzzling.

On the whole, the papers on synaptogenesis are the weakest, because of their limited scope and their failure to provide enough background material to make the data presented meaningful to those other than retinal synaptologists. Some of the papers are little more than summaries of work produced by one laboratory. The first paper in this set, by S. C. Sharma, is an exception in that it reviews one of the more controversial issues in the field of retinotectal connectivity, the polarization of retina axes and the specification of retinal ganglion cells during embryonic development. Sharma describes the original proposal of $M$. Jacobson and R. K. Hunt that the axes of the retina are not fixed until embryonic stages 28-30 in Xenopus laevis, at which time the retinal ganglion cells acquire a specific "label" or an "address" which thereafter governs their choice of termination site in the optic tectum. Attempts by Sharma and colleagues and by R. M. Gaze and colleagues to repeat this work were unsuccessful. An elegant experiment recently published by $\mathrm{C}$. Holt demonstrated that a previously unsuspected translocation of cells takes place during morphogenesis of the optic cup, and it was recognized that these cellular shifts could explain some of the conflicting data. If nothing else, this account provides a disquieting tale of how scientists can be misled into constructing elaborate theories based on what prove to be artifactual results.

The pair of papers on intercellular communication are the best of the lot, even though they have the least to do with ocular or visual development. M. V. L. Bennett and colleagues have written a comprehensive and authoritative review of the literature on gap junctions. Included are concise discussions of the structure, the biochemistry, the physiology and the possible roles that these channels of direct intercellular communication might play in development. Curiously, despite the ubiquity of these structures in embryos and the widespread recognition that they must be playing some important role, there is no direct evidence about what that role might be. A paper by J. A. Raper and C. S. Goodman describes an interesting series of events in developing grasshopper embryos. At early embryonic stages many cells are dye-coupled, but as development progresses, coupling becomes increasingly restricted to specific patterns of cells that are related to each other either by lineage or by position. The nature of the structure mediating this interaction is uncertain, although the gap junction is a likely possibility. Of particular interest is the observation that young, growing axons become coupled to cells along their pathway, via the specialized tip of their outgrowing process, the growth cone. Such selective interactions might be significant in guiding axons to their specific targets, a subject of much current research interest in developmental neurobiology.

The third topic is represented by a group of three papers about humoral factors and their involvement in certain morphogenetic and pathogenetic ocular events. One paper describes the work of the Laboratory for Anatomy and Embryology in Utrecht on the inductive influence of the eye cup in the morphogen- 
esis of the lens. The paper is an historical, biographical account of the efforts of this laboratory, and of others, to identify the inductor substance. Some progress has been made in characterizing the inductor, which appears to be a small, trypsin-sensitive molecule, but little else is known about it.

Another paper concerns the effects of insulin-like mitogens on lens morphogenesis and cataract pathogenesis. It is similarly biographical in style and is an interesting account of the discovery of the role of somatomedin in controlling mitosis in lens epithelium. Somatomedin is a peptide whose synthesis in the liver is regulated by the concentration of growth hormone (somatotropin), whose effects it mediates. The discovery that mitotic activity in the lens epithelium is regulated by somatomedin has allowed these workers to provide a direct demonstration in support of the theory that mitosis is the force which drives the movement of lens cells into the area where lens fiber generation takes place. The relation of mitosis to radiation cataracts was also demonstrated with similar experiments in which hormonal factors were manipulated.

The last paper about humoral factors is a methodological and clinical summary of studies on a retinaderived angiogenic factor that may be important in the pathology of retinal neovascularization. The angiogenic factor in retinal extracts is a protein of molecular weight $50,000-100,000$ daltons. Its effect in promoting growth of blood vessels is, under normal conditions, probably counteracted by an anti-angiogenic factor found in the vitreous.

From the above brief description it should be clear that the spectrum of topics covered in this book is quite broad. Most have in common only their association with ocular development, but there are exceptions even to that. Nevertheless, this volume will be of value to those who seek a quick exposure to some of the latest ideas and issues surrounding the concept that cells exchange information with one another in the developing organism.

\section{Pamela A. Raymond}

Department of Anatomy and Cell Biology

University of Michigan Medical School

Ann Arbor, Michigan 48109

\section{Developing Neurobiology}

\author{
Genetic Approaches to Developmental \\ Neurobiology. \\ Edited by Y. Tsukada. \\ New York: Springer-Verlag. (1982). 269 pp. \$43.00.
}

This is a compendium of papers given at a symposium in Tokyo, in May 1981. The stated aim of the symposium was to "understand the organogenesis of the nervous system, particularly the early development which is under genetic control" (p. xiii). As a developmental neurobiologist, though not expert in any of the fields covered in this book, I was quite disappointed by it. For the most part, it illustrates the problems associated with publications that simply sketch symposium proceedings.

The book is divided into three sections: early development of mammals; genetics of mammalian embryos; and mammalian mutants. Not covered are topics most readers might expect, such as invertebrate neuronal cell lineage analysis, neuronal connectivity and the genetics of proteins associated with neurotransmission. Furthermore, all three sections suffer from the same major problems-lack of any statement of intent and lack of consistency among individual chapters. Prefatory notes for each section would have helped tremendously, and a strong editorial policy delineating expectations for each section would have improved the presentations substantially. As it is, chapters vary widely from short summaries to discussions of primary and, in some cases, unsubstantiated data.

In the presentations devoted to early development of mammals, only the chapter by R. S. P. Beddington has concise background material preceding her description of mammalian cell lineages. Of course, one of the major experimental problems in this area is the difficulty of manipulating populations of cells in these embryos, at least as compared with avian embryos (as so elegantly illustrated by the work of N. Le Douarin, presented later in this book). Other chapters focus on in vitro fertilization and development of mammalian embryos, but are concerned primarily with technical matters, such as appropriate media for carrying out successful fertilizations.

In the section on genetics of mammalian embryos is a chapter by Okada et al. discussing $\mathrm{Ca}^{2+}$-independent and -dependent mechanisms of cell-to-cell (primarily fibroblasts and embryonal carcinoma cells) aggregation. There are two chapters on the production of teratocarcinomas-one by L. C. Stevens, devoted to a genetic analysis of the origin of teratocarcinogenesis, and one by T. Noguchi et al., who describe a reliable way of producing these tumors following inguinal subcutaneous injection of embryonal carcinoma cells. Two articles, by Onsawa and by K. Yamamura, describe the use of mammalian chimeras to study the genetics of pigmentation patterns. The best article in this section on mammalian embryos is that by $N$. Le Douarin briefly describing her cell lineage analysis of parasympathetic and sympathetic ganglia neurons in chick-quail chimeras. Although she has reported on this work more completely in other primary publications and reviews, she is still one of the few authors in this book to set out her experimental aims, outline her results and state her conclusions clearly. 